\title{
Robust Adaptive Path Following Control of an Unmanned Surface Vessel Subject to Input Saturation and Uncertainties
}

\author{
Yunsheng Fan * D , Hongyun Huang and Yuanyuan Tan \\ School of Marine Electrical Engineering, Dalian Maritime University, Dalian 116026, China; \\ iamhongyun@gmail.com (H.H.); tanyuanyuan@dlmu.edu.cn (Y.T.) \\ * Correspondence: yunsheng@dlmu.edu.cn
}

Received: 17 February 2019; Accepted: 25 April 2019; Published: 1 May 2019

check for updates

\begin{abstract}
This paper investigates the path following control problem of an unmanned surface vessel (USV) subject to input saturation and uncertainties including model parameters uncertainties and unknown time-varying external disturbances. A nonlinear robust adaptive control scheme is proposed to address the issue, more specifically, steering a USV to follow the desired path at a certain velocity assignment despite the involved disturbances, by utilizing the finite-time currents observer based line-of-sight (LOS) guidance and radial basis function neural networks (RBFNN). Backstepping and Lyapunov's direct method are the main design frameworks. Based on the finite-time currents observer and adaptive control technique, an improved LOS guidance law is proposed to obtain the desired approaching angle to the desired path, making compensations for the effects of unknown time-varying ocean currents. Then, a kinetic controller with the capability of uncertainties estimation and disturbances rejection is proposed based on the RBFNNs, where the adaptive laws including leakage terms estimate the approximation error and the unknown time-varying disturbances. Subsequently, sophisticated auxiliary control systems are employed to handle input saturation constraints of actuators. All error signals of the closed-loop system are proved to be locally uniformly ultimately bounded (UUB). Numerical simulations demonstrated the effectiveness and robustness of the proposed path following control method.
\end{abstract}

Keywords: unmanned surface vessel; path following; integral line-of-sight; finite-time currents observer; radial basis function neural networks; input saturation

\section{Introduction}

Unmanned surface vessel (USV) as an intelligent and autonomous marine equipment has received more and more attention from the control community, for broad application in the cluttered ocean environment, especially in cases where human intervention is not possible [1]. Generally speaking, three different types of control technologies play a crucial role in the development of USVs: path following control, trajectory tracking control and set-point control [2]. Many researchers propose lots of relevant control strategies and address the issues to a various extent. This study continues along the works and contributions of the predecessors. This paper aims at the path following control, mainly discussing the guidance and control of a USV. Path following is usually defined as steering a vessel to follow the desired path at a certain speed, which is not specified with temporal constraint [3]. Although there are considerable theoretical studies regarding the path following and practical engineering achievements, practical studies of the path following control for USVs have progressed haltingly amid great difficulties. It is essential to develop a highly accurate and robust path following controller for a USV when executing various vital missions. Therefore, under the circumstance of severe sea state, the safe operation and mission execution can be guaranteed. 
Two aspects play crucial roles in the path following control scheme: guidance and control [4]. Guidance can refer to the popular and effective line-of-sight (LOS) guidance, refining missile guidance approach or marine guidance $[5,6]$. This special guidance law exploits the geometry relationships to generate a yaw angle known as the approaching angle, which is fed into the control system. In other words, the control system tracks the reference yaw angle signal together with the specified velocity tracking. Hence, the performance of the path following heavily depends on the guidance system. It turns out that classic LOS guidance is simple and effective [7], albeit with limitations in the case of being exposed to the complex ocean environment induced by waves, wind, and ocean currents. Moreover, the traditional LOS guidance will cause large cross-tracking error when the marine surface vehicles are in steady state, which strictly depends on the path curvature and the magnitude of the drift force. Therefore, the traditional LOS was extended to various forms such as integral LOS (ILOS) guidance and adaptive LOS (ALOS) guidance by Fossen. In [8], an ILOS guidance with integral action is proposed to handle the constant and irrotational ocean currents and other environmental disturbances including wind or waves. In [9], a new ILOS with time-varying lookahead distance is presented with the capability of canceling the effects of constant environmental disturbances, like constant ocean currents. In [10], the direct and indirect adaptive ILOS based path following controller is proposed to deal with the ocean currents. Another way to improve the LOS guidance is to estimate the sideslip angle caused by the external disturbance as an observer-based strategy. In [11], a novel adaptive LOS (ALOS) guidance with small computation footprint is proposed where the adaptive laws dominate the sideslip angle compensation rate. The sideslip angle is treated as an unknown constant, which significantly limits the application of ALOS. In [12], a reduced-order extended state observer (ESO) based LOS guidance is proposed to deal with the time-varying sideslip angle, which is appropriate for straight-line and curved path following. In [13], the magnitude and convergence speed of the sideslip angle is considered, which can be identified by the constructed finite-time sideslip angle observer. However, on the one hand, it should be noted that the aforementioned literature (e.g., [8-10]) merely solve the problem in the kinematic level, i.e., ignoring the along-tracking error. On the other hand, the method in [10] only adds the ocean currents in the kinematic model in terms of the relative surge and sway velocities, whereas the along-tracking error is omitted.

The dynamics control system as the execution system of a USV is another crucial constituent in the path following scheme. In general, USVs do not have an independent actuator in their sway direction. This nonholonomic constraint and underactuated nature makes the control system design much more challenging [14]. To satisfy the successful execution of missions and achieve expected performance and robustness in the full range of work space from calm ocean environment to severe ocean environment along with inaccuracy system parameters, various nonlinear control methods have been proposed [15-22]. In [15], external disturbances rejection method based on the reduced-order linear ESO is proposed. In [16], Do proposed a global path-tracking controller for underactuated ships under deterministic and stochastic loads where weak nonlinearly and strong Lyapunov design method is introduced and the estimations of disturbances are updated by projection algorithms. In [17,18], nonlinear disturbance observers are utilized to estimate the ubiquitous external disturbances in the dynamics model of marine vehicles, where the disturbance estimation errors are proved to be UUB. In [19], a nonlinear adaptive PI sliding mode tracking controller is proposed to solve the environmental disturbance problems by relaxing the assumption of knowing the upper bound of the disturbance. In $[20,21]$, a novel adaptive switching-gain-based control method is proposed for a general uncertain Euler-Lagrange system, which is not only insensitive to the nature of uncertainties but can also alleviate the overestimation-underestimation problem. Moreover, intelligent control such as fuzzy and neural control has been applied to deal with the uncertainties and disturbances of underactuated marine surface ships. In [22], Wang proposed an adaptive online constructive fuzzy controller where the fuzzy approximator is used to approximate the unknown disturbances. Besides, robust radial basis function neural network control laws and iterative neural network control laws are proposed in [23,24], respectively, which are devoted to identifying and compensating the dynamical uncertainties and 
external disturbances. In addition, it should be noted that few of the aforementioned path following control schemes take into account input saturation. In fact, the practical constraint of actuators determined by the maximum forces and moments would degrade the performance of the control system or even make it unstable. Therefore, it is essential to implement the emulate of the constraint on the control laws design process for reliability and robustness.

Motivated by the observations and considerations mentioned above, a finite-time currents observer based ILOS guidance is proposed to deal with unknown time-varying ocean currents, which is suitable for any desired parametric path with high accuracy control performance. Subsequently, adaptive control laws based on the RBFNN are designed, which solve input saturation with sophisticated auxiliary systems simultaneously. A robust adaptive controller is developed to address the path following problem, which is verified to be effective via numerical simulations. The main contributions of the paper are summarized as follows.

(1) A finite-time currents observer based LOS guidance is presented to obtain the desired yaw angle and estimate the unknown time-varying ocean currents precisely, which significantly influences the performance of the control subsystem.

(2) The RBF neural networks are incorporated into the kinetic controller to solve the uncertainties, which does not require any prior knowledge of the dynamics of the USV and disturbances, and the adaptive laws are designed to estimate the compound bounds of approximating errors and external time-varying disturbances.

(3) The input constraint effect is analyzed with auxiliary systems and the states of auxiliary systems are utilized to make compensations for input saturation, which attenuates the challenge of the actuators.

The rest sections are organized as follows. Section 2 presents the preliminaries and problem formulation. Section 3 provides the design details of the guidance, the kinetic controller, and the stability analysis. Simulation results are presented and analyzed in Section 4. Finally, Section 5 concludes the paper.

\section{Preliminaries and Problem Formulation}

\subsection{RBFNN Approximation}

Consider an unknown smooth nonlinear function $f(\mathbf{x}): \mathbb{R}^{m} \rightarrow \mathbb{R}$ can be approximated on a compact set $\Omega \in \mathbb{R}^{m}$ by the following RBFNN:

$$
f(\mathbf{x})=\mathbf{W}^{* T} \boldsymbol{\varphi}(\mathbf{x})+\varepsilon
$$

where $\mathbf{x} \in \Omega$ is the input vector, $\varepsilon$ is the approximation error and satisfies $|\varepsilon| \leqslant \bar{\varepsilon}$ and $\bar{\varepsilon}$ is a constant, and the node number of the $\mathrm{NN}$ is $l>1 . \mathbf{W}^{*} \in \mathbb{R}^{l}$ represents the optimal weight vector, which is defined by

$$
\mathbf{W}^{*}=\arg \min _{\hat{\mathbf{W}} \in R^{l}}\left\{\sup _{\mathbf{x} \in \mathbf{\Omega}}\left|f(\mathbf{x})-\hat{\mathbf{W}}^{T} \boldsymbol{\varphi}(\mathbf{x})\right|\right\}
$$

where $\hat{\mathbf{W}}$ is the estimation of $\mathbf{W}^{*} . \boldsymbol{\varphi}(\mathbf{x})=\left[\varphi_{1}, \varphi_{2}, \ldots, \varphi_{l}\right]^{T}: \Omega \rightarrow \mathbb{R}^{l}$ represents the radial function vector, the element of which is chosen as the Gaussian function:

$$
\varphi_{i}(\mathbf{x})=\exp \left(-\frac{\left(x-b_{i}\right)^{T}\left(x-b_{i}\right)}{c_{i}{ }^{2}}\right)(i=1,2, \ldots, l)
$$

where $\mathbf{b}=\left[b_{1}, b_{2}, \ldots, b_{l}\right]^{T}$ and $\mathbf{c}=\left[c_{1}, c_{2}, \ldots, c_{l}\right]^{T}$ are the centers of receptive field and spread of the Gaussian function, respectively. 


\subsection{USV Model}

As illustrated in Figure 1, the position and orientation describe the horizontal plane motion of a USV neglecting roll, pitch, and heave, where $\{i\}$ and $\{b\}$ represent the inertial frame and body fixed frame, respectively. The desired continuous path $P_{d}(\theta)=\left[x_{d}(\theta), y_{d}(\theta)\right]^{T}$ is parameterized by a scalar variable $\theta$ and $P=[x, y]^{T}$ is the position coordinate. The kinematic equations of a USV can be described by relative velocities as follows [23]

$$
\left\{\begin{array}{l}
\dot{x}=u_{r} \cos \psi-v_{r} \sin \psi+V_{x} \\
\dot{y}=u_{r} \sin \psi+v_{r} \cos \psi+V_{y} \\
\dot{\psi}=r
\end{array}\right.
$$

where $u_{r}$ and $v_{r}$ are the relative surge and sway velocities; $x, y$, and $\psi$ express the position and orientation in $\{i\}$; and $V_{x}$ and $V_{y}$ are the ocean currents represented in $\{b\}$. The dynamics of a USV is expressed as follows [25]

$$
\left\{\begin{array}{l}
\dot{u}_{r}=\underbrace{}_{f_{u}} \underbrace{\frac{m_{22}}{m_{11}} v_{r} r-\frac{d_{11}}{m_{11}} u_{r}-\sum_{i=2}^{3} \frac{d_{u i}}{m_{11}}\left|u_{r}\right|^{i-1} u_{r}}_{f_{v}}+\frac{1}{m_{11}} \tau_{u}+\frac{1}{m_{11}} \tau_{w u} \\
\dot{v}_{r}=\underbrace{-\frac{m_{11}}{m_{22}} u_{r} r-\frac{d_{22}}{m_{22}} v_{r}-\sum_{i=2}^{3} \frac{d_{v i}}{m_{11}}\left|v_{r}\right|^{i-1} v_{r}}_{f_{r}}+\frac{1}{m_{22}} \tau_{w v} \\
\dot{r}=\underbrace{\frac{\left(m_{11}-m_{22}\right)}{m_{33}} u_{r} v_{r}-\frac{d_{33}}{m_{33}} r-\sum_{i=2}^{3} \frac{d_{r i}}{m_{11}}|r|^{i-1} r}_{i=2}+\frac{1}{m_{33}} \tau_{r}+\frac{1}{m_{33}} \tau_{w r}
\end{array}\right.
$$

where the positive constant parameters $m_{j j}(j=1,2,3)$ are the inertia including added mass; $d_{i i}, d_{u i}, d_{v i}$, and $d_{r i}(i=2,3)$ are the linear and quadratic hydrodynamic damping in surge, sway, and yaw; $\tau_{w u}, \tau_{w v}$, and $\tau_{w r}$ are the unknown time-varying environmental disturbances; and $\tau_{u}$ and $\tau_{r}$ are the available control inputs of the surge force and the yaw moment thereby viewing it as the underactuated control problem. Since the model parameters are directly related to the operation conditions [26], the parameters of the model is uncertain. Actually, due to the physical constraint, the control inputs are subject to nonlinear saturations, which is shown as follows

$$
\tau_{i}= \begin{cases}\tau_{i \max }, & \tau_{i 0}>\tau_{i \max } \\ \tau_{i 0}, & \tau_{i \min } \leqslant \tau_{i 0} \leqslant \tau_{i \max } \\ \tau_{i \min }, & \tau_{i 0}<\tau_{i \min }\end{cases}
$$

where $\tau_{i \min }$ and $\tau_{i \max }(i=u, r)$ are the minimum and maximum control inputs produced by the actuators, referring to actual constraints of the motor's rotational speed and rudder deflection; and $\tau_{i 0}$ is the command control input of the path following controller.

Assumption 1. Assume that all states of a USV are measurable.

Assumption 2. The time-varying ocean currents $v=\left[V_{x}, V_{y}\right]^{T}$ are assumed to be irrotational and bounded, and there exist a positive constant $M$, such that $\|\dot{v}\| \leqslant M, M>0$. The disturbances $\tau_{w i}(i=u, v, r)$ are unknown time-varying and bounded, and the first derivative of them are also bounded such that $\left|\dot{\tau}_{w i}\right| \leqslant \bar{\tau}_{w}$, where $\bar{\tau}_{w}$ is unknown constant. 
Remark 1. Note that in (5) the off-diagonal terms of the inertia and damping are ignored. No matter a large scale surface vessel or a highly maneuverable unmanned surface vessel, these terms are relatively small than the main diagonal terms. Therefore, it is reasonable to omit these terms.

Remark 2. The external disturbances $\tau_{w u}, \tau_{w v}$, and $\tau_{w r}$ in Equation (5) represent the compound disturbances of the wind and wave disturbances. The ocean currents as the form of hydrodynamic terms with relative velocities are represented in Equation (4).

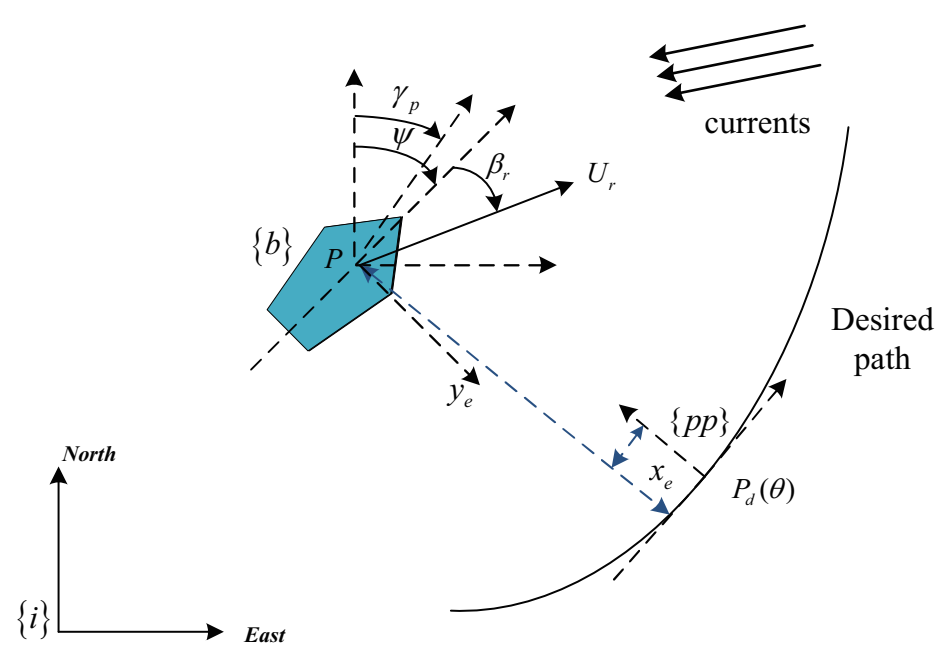

Figure 1. USV path following guidance information illustration.

\subsection{Control Objective}

In Figure 1, a local path parallel reference frame is denoted as $\{p p\}$. To arrive at $\{p p\},\{i\}$ should be rotated an angle $\gamma_{p}(\theta)=\operatorname{atan} 2\left(y_{d}^{\prime}(\theta), x_{d}^{\prime}(\theta)\right)$, where the notation $y_{d}^{\prime}(\theta)=d y_{d}(\theta) / d \theta$ is used. Therefore, the position errors can be given as follows

$$
\left[\begin{array}{l}
x_{e} \\
y_{e}
\end{array}\right]=\underbrace{\left[\begin{array}{cc}
\cos \gamma_{p} & -\sin \gamma_{p} \\
\sin \gamma_{p} & \cos \gamma_{p}
\end{array}\right]^{T}}_{\mathbf{R}^{\mathrm{T}}\left(\gamma_{p}\right)}\left(P-P_{d}(\theta)\right)
$$

where $x_{e}$ and $y_{e}$ are the along-tracking error and cross-tracking error, respectively, and $\mathbf{R}\left(\gamma_{p}\right)$ is the rotation matrix. Meanwhile, we also have

$$
\dot{P}_{d}(\theta)=\mathbf{R}\left(\gamma_{p}\right)\left[U_{p p}, 0\right]^{T}
$$

where $\left[U_{p p}, 0\right]^{T}$ is the velocity of $\{p p\}$ with respect to $\{i\}$, represented in $\{p p\}$.

The path following control problem is concerned with designing control laws to reach and then keep following the desired path. Once the path is reached, the vessel can maintain a desired surge velocity assignment of $u_{d}$. It is worth noting that a constant speed profile is frequently chosen in many cases. Therefore, the control objective is as follows:

$$
\sup _{t>0}\left|x_{e}\right| \leqslant \delta_{1}, \sup _{t>0}\left|y_{e}\right| \leqslant \delta_{2}, \sup _{t>0}\left|u_{r}-u_{d}\right| \leqslant \delta_{3}
$$

where $\delta_{1}, \delta_{2}$, and $\delta_{3}$ are small positive constants. Meanwhile, it is guaranteed that all error signals of the closed-loop system are locally UUB. 
Assumption 3. The desired path should be sufficiently smooth such that its first derivative $\dot{P}_{d}$ is bounded. In addition, the desired speed assignment $u_{d}$ and its first derivative are bounded.

\section{Main Results}

This section presents the design details of the path following controller to satisfy the control objective that concluded in Section 2. The whole controller consists of two parts: the modified ILOS guidance module and the kinetic controller, which is shown in Figure 2. The ILOS guidance is utilized to calculate the desired yaw angle, where the constructed finite-time currents observer can provide the fast and precise estimation of the unknown time-varying ocean currents. By incorporating the RBFNNs into the backstepping design method, the kinetic controller are developed. Finally, the stability analysis is presented to validate the feasibility of the proposed control approach.

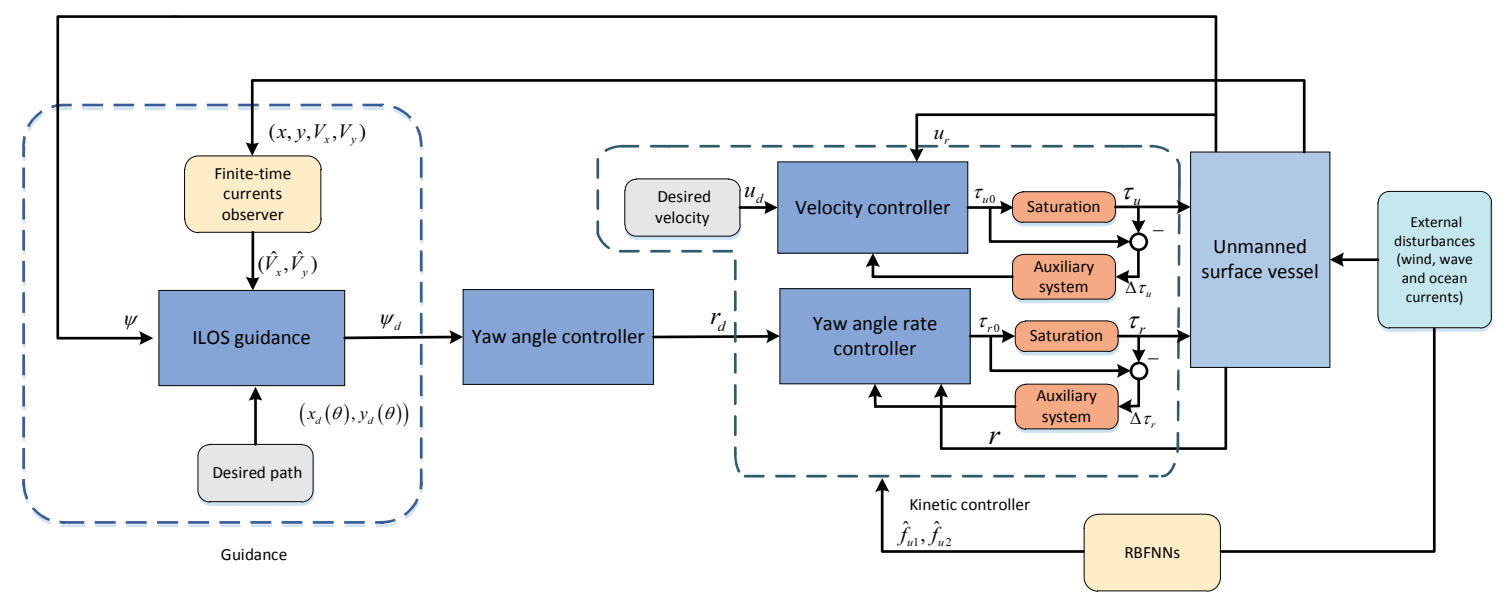

Figure 2. The structure diagram of the path following controller.

\subsection{Guidance}

This section is devoted to designing the ILOS guidance to calculate the approaching angle with respect to the desired path. Then, the time derivative of Equation (7) is taken to obtain the position errors dynamics

$$
\left\{\begin{array}{l}
\dot{x}_{e}=u_{r} \cos \left(\psi-\gamma_{p}\right)-v_{r} \sin \left(\psi-\gamma_{p}\right)+y_{e} \dot{\gamma}_{p}+\theta_{x}-u_{p p} \\
\dot{y}_{e}=u_{r} \sin \left(\psi-\gamma_{p}\right)+v_{r} \cos \left(\psi-\gamma_{p}\right)-x_{e} \dot{\gamma}_{p}+\theta_{y}
\end{array}\right.
$$

where $\theta_{x}=V_{c} \cos \left(\beta_{c}-\gamma_{p}\right)$ and $\theta_{y}=V_{c} \sin \left(\beta_{c}-\gamma_{p}\right) ; V_{c}=\sqrt{V_{x}^{2}+V_{y}^{2}} \leqslant V_{c \max }$ and $\beta_{c}=\operatorname{atan} 2\left(V_{y}, V_{x}\right)$. By using the kinematic relationship given by Equation (8), we have $\dot{\theta}=U_{p p} / \sqrt{\dot{x}_{d}^{2}+\dot{y}_{d}^{2}}$. In addition, the virtual target velocity $U_{p p}$ can be regarded as the extra degree-of-freedom to stabilize the cross-tracking error [5]. To prescribe the desired yaw angle $\psi_{d}$ for $\psi$, the following form of the guidance law is taken

$$
\psi_{d}=\gamma_{p}-\beta_{r}+\operatorname{atan} 2\left(-\left(y_{e}+\alpha_{y}\right), \Delta\right)
$$

where $\beta_{r}=\operatorname{atan} 2\left(v_{r}, u_{r}\right) ; \Delta$ is the specific look-ahead distance; and $\alpha_{y}$ is the virtual control input. It should be noted that $\alpha_{y}$ is introduced to shape the dynamics of the ILOS guidance by inherently adding an integral action.

\subsubsection{Estimations of Ocean Currents}

Before designing the kinematic controller, the unknown ocean currents need be identified precisely. The finite-time currents observer for $V_{x}$ and $V_{y}$ are designed as follows: 


$$
\left\{\begin{array}{l}
\dot{\hat{\varepsilon}}_{e}=\boldsymbol{\Lambda}+\mathbf{g} \\
\boldsymbol{\Lambda}=-\rho_{1} \mathbf{L}^{1 / 2} \operatorname{sig}^{1 / 2}\left(\hat{\varepsilon}_{e}-\varepsilon_{e}\right)+\hat{v} \\
\dot{\hat{v}}=-\rho_{2} \mathbf{L} \operatorname{sig}(\hat{v}-\Lambda)
\end{array}\right.
$$

where $\operatorname{sig}^{\alpha}(\bullet)=|\bullet|^{\alpha} \operatorname{sign}(\bullet) ; \hat{\varepsilon}_{e}=[\hat{x}, \hat{y}]^{T} ; \mathbf{g}=\left[U_{r} \cos \left(\psi+\beta_{r}\right), U_{r} \sin \left(\psi+\beta_{r}\right)\right]^{T}, U_{r}=\sqrt{u_{r}^{2}+v_{r}^{2}}$; $\hat{v}=\left[\hat{V}_{x}, \hat{V}_{y}\right]^{T} ; \mathbf{L}=\operatorname{diag}\left(l_{1}, l_{2}\right)>0 ; \rho_{1}>0, \rho_{2}>0$.

Theorem 1. Considering the proposed finite-time currents observer in Equation (12), the unknown time-varying ocean currents $\boldsymbol{v}$ can be precisely estimated within a finite time.

Proof. According to Equation (4), we have

$$
\dot{\varepsilon}_{e}=\mathbf{g}+v
$$

Together with the finite-time currents observer, we have

$$
\left\{\begin{aligned}
\dot{\tilde{\varepsilon}}_{e} & =-\rho_{1} \mathbf{L}^{1 / 2} \operatorname{sig}^{1 / 2}\left(\hat{\boldsymbol{\varepsilon}}_{e}-\boldsymbol{\varepsilon}_{e}\right)+\tilde{\boldsymbol{v}} \\
\dot{\tilde{v}} & =-\rho_{2} \mathbf{L} \operatorname{sig}(\hat{\boldsymbol{v}}-\boldsymbol{\Lambda})-\dot{\boldsymbol{v}} \\
& \in-\rho_{2} \mathbf{L} \operatorname{sig}(\hat{\boldsymbol{v}}-\boldsymbol{\Lambda})+[-M, M]
\end{aligned}\right.
$$

where $\tilde{\varepsilon}_{e}=\hat{\varepsilon}_{e}-\varepsilon_{e}$ and $\tilde{v}=\hat{v}-v$.

In light of Lemma 2 in [27], one can immediately have observer errors $\tilde{\varepsilon}_{e}$ and $\tilde{v}$ converge to zero within a finite time. It implies that there exists a finite time $T_{0}$ and finite constants $\varepsilon_{b}$ and $v_{b}$ such that

$$
\left\{\begin{array}{l}
\left\|\tilde{\varepsilon}_{e}\right\|<\varepsilon_{b},\|\tilde{\boldsymbol{v}}\|<v_{b}, \forall t<T_{0} \\
\tilde{\varepsilon}_{e} \equiv 0, \tilde{\boldsymbol{v}} \equiv 0, \forall t \geqslant T_{0}
\end{array}\right.
$$

This concludes the proof.

Therefore, the parametric currents estimation errors $\tilde{\theta}_{x}=\theta_{x}-\hat{\theta}_{x}$ and $\tilde{\theta}_{y}=\theta_{y}-\hat{\theta}_{y}$ satisfy the following inequality

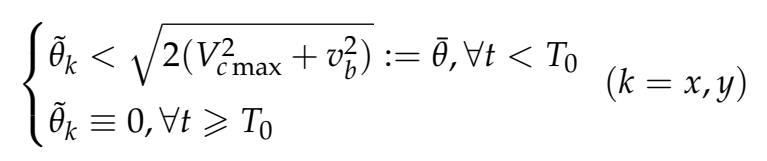

Remark 3. With the availability of $\hat{V}_{x}$ and $\hat{V}_{y}$ of the finite-time currents observer, the estimations of parametric currents $\hat{\theta}_{x}$ and $\hat{\theta}_{y}$ can be reliably obtained.

\subsubsection{Design of Kinematic Controller}

In this section, the kinematic control laws including the virtual control law and path variable update law are presented.

Substituting Equation (11) into Equation (10) results in

$$
\left\{\begin{array}{l}
\dot{x}_{e}=U_{r} \cos \left(\psi-\gamma_{p}+\beta_{r}\right)+\theta_{x}-U_{p p}+y_{e} \dot{\gamma}_{p} \\
\dot{y}_{e}=-\frac{U_{r}\left(y_{e}+\alpha_{y}\right)}{\sqrt{\left(y_{e}+\alpha_{y}\right)^{2}+\Delta^{2}}}+U_{r} \phi\left(y_{e}, \tilde{\psi}\right) \tilde{\psi}+\theta_{y}-x_{e} \dot{\gamma}_{p}
\end{array}\right.
$$

where $\tilde{\psi}=\psi-\psi_{d}$ and $\phi\left(y_{e}, \tilde{\psi}\right)=\frac{\sin \tilde{\psi}}{\tilde{\psi}} \frac{\Delta}{\sqrt{\left(y_{e}+\alpha_{y}\right)^{2}+\Delta^{2}}}-\frac{\cos \tilde{\psi}-1}{\tilde{\psi}} \frac{\left(y_{e}+\alpha_{y}\right)}{\sqrt{\left(y_{e}+\alpha_{y}\right)^{2}+\Delta^{2}}}$. Note that the upper bound of $\phi\left(y_{e}, \tilde{\psi}\right)$ is 1.73 . 
The virtual control $\alpha_{y}$ is designed to cancel $\theta_{y}$ asymptotically [10]

$$
\frac{U_{r} \alpha_{y}}{\sqrt{\left(y_{e}+\alpha_{y}\right)^{2}+\Delta^{2}}}=\hat{\theta}_{y}
$$

Solving for $\alpha_{y}$ provides a credible solution (the negative root) as follows

$$
\alpha_{y}=\frac{y_{e}\left(\hat{\theta}_{y} / U_{r}\right)^{2}-\hat{\theta}_{y} / U_{r} \sqrt{\Delta^{2}\left(1-\left(\hat{\theta}_{y} / U_{r}\right)^{2}\right)+y_{e}^{2}}}{1-\left(\hat{\theta}_{y} / U_{r}\right)^{2}}
$$

In this case, the boundedness of $\hat{\theta}_{y} / U_{r}$ must be ensured such that $\left|\hat{\theta}_{y} / U_{r}\right|<1$. Thus, the boundedness of the parametric ocean currents is defined such that $\theta_{y}<M_{\theta}<U_{r}$.

The desire target velocity $U_{p p}$ is chosen to stabilize $x_{e}$ as follows

$$
U_{p p}=U_{r} \cos \left(\psi-\gamma_{p}+\beta_{r}\right)+k_{1} x_{e}+\hat{\theta}_{x}
$$

where $k_{1}$ is a positive design parameter. Thus, the path variable update law can be obtained according to Equation (8):

$$
\dot{\theta}=\frac{U_{p p}}{\sqrt{\dot{x}_{d}^{2}+\dot{y}_{d}^{2}}}
$$

Therefore, substituting Equations (18) and (19) into Equation (17), we have

$$
\left\{\begin{array}{l}
\dot{x}_{e}=-k_{1} x_{e}+\tilde{\theta}_{x}+y_{e} \dot{\gamma}_{p} \\
\dot{y}_{e}=-\frac{U_{r} y_{e}}{\sqrt{\left(y_{e}+\alpha_{y}\right)^{2}+\Delta^{2}}}+U_{r} \phi\left(y_{e}, \tilde{\psi}\right) \tilde{\psi}+\tilde{\theta}_{y}-x_{e} \dot{\gamma}_{p}
\end{array}\right.
$$

\subsection{Design of Kinetic Controller}

In this section, a kinetic controller is designed to track the desired approaching angle and the desired surge velocity. Let $\tilde{r}=r-r_{d}$ and $\tilde{u}_{r}=u_{r}-u_{d}$ be the attitude tracking error and surge velocity tracking error, respectively, where $r_{d}$ is the virtual control law and $u_{d}$ is the desired surge velocity.

According to Equation (4), the time derivative of $\tilde{\psi}$ is given by

$$
\dot{\tilde{\psi}}=r-\dot{\psi}_{d}
$$

To stabilize Equation (23), the desired intermediate control law for $r_{d}$ is chosen as"

$$
r_{d}=-k_{2} \tilde{\psi}+\dot{\psi}_{d}-U_{r} \phi y_{e}
$$

where $k_{2}$ is a positive design parameter.

From Equation (5), the dynamics of $\tilde{r}$ and $\tilde{u}_{r}$ are given as follows

$$
\left\{\begin{array}{c}
m_{33} \dot{\tilde{r}}=m_{33} f_{r}+\tau_{r}+\tau_{w r}-m_{33} \dot{r}_{d} \\
m_{11} \dot{\tilde{u}}_{r}=m_{11} f_{u}+\tau_{u}+\tau_{w u}-m_{11} \dot{u}_{d}
\end{array}\right.
$$

Since the inertia and damping parameters are unknown, RBFNNs are employed to handle the unknown parts

$$
\left\{\begin{aligned}
m_{33} f_{r}-m_{33} \dot{r}_{d} & =\mathbf{W}_{1}^{* T} \boldsymbol{\varphi}(\mathbf{z})+\varepsilon_{1} \\
m_{11} f_{u}-m_{11} \dot{u}_{d} & =\mathbf{W}_{2}^{* T} \boldsymbol{\varphi}(\mathbf{z})+\varepsilon_{2}
\end{aligned}\right.
$$


where $\mathbf{W}_{i}^{*}(i=1,2)$ is the ideal constant weight matrix satisfying $\left\|\mathbf{W}_{i}^{*}\right\| \leqslant W_{i M} ; \boldsymbol{\varphi}(\mathbf{z})$ is the radial basis function with $\mathbf{z}=\left[u_{r}, v_{r}, r\right]^{T}$ being the input vector to the RBFNNs; and $\varepsilon_{i}(i=1,2)$ is the approximation error with unknown constant upper bound such that $\left|\varepsilon_{i}\right| \leqslant \bar{\varepsilon}_{i}$. Denote the unknown parts as $m_{33} f_{r}-m_{33} \dot{r}_{d}=f_{u 1}$ and $m_{11} f_{u}-m_{11} \dot{u}_{d}=f_{u 2}$. Furthermore, there exists bounded functions $\delta_{1}$ and $\delta_{2}$ such that $\left|\varepsilon_{1}\right|+\left|\tau_{w r}\right| \leqslant \delta_{1}$, and $\left|\varepsilon_{2}\right|+\left|\tau_{w u}\right| \leqslant \delta_{2}$.

Therefore, the nominal control inputs are chosen as follows by considering the input saturation

$$
\left\{\begin{array}{l}
\tau_{r 0}=-k_{3} \tilde{r}-\tilde{\psi}-\hat{\mathbf{W}}_{1} \boldsymbol{\varphi}(\mathbf{z})-\hat{\delta}_{1} h(\tilde{r})+k_{\zeta 1} \sigma_{1} \\
\tau_{u 0}=-k_{4} \tilde{u}_{r}-\hat{\mathbf{W}}_{2} \boldsymbol{\varphi}(\mathbf{z})-\hat{\delta}_{2} h\left(\tilde{u}_{r}\right)+k_{\zeta 2} \sigma_{2}
\end{array}\right.
$$

with update laws

$$
\begin{gathered}
\left\{\begin{array}{c}
\dot{\mathbf{W}}_{1}=\Gamma_{1}\left(\boldsymbol{\varphi}(\mathbf{z}) \tilde{r}-\iota_{1} \hat{\mathbf{W}}_{1}\right) \\
\dot{\hat{\delta}}_{1}=\xi_{1}\left(\tilde{r} h(\tilde{r})-\lambda_{1}\left(\hat{\delta}_{1}-\delta_{1}^{0}\right)\right)
\end{array}\right. \\
\left\{\begin{array}{c}
\dot{\hat{\mathbf{W}}}_{2}=\Gamma_{2}\left(\boldsymbol{\varphi}(\mathbf{z}) \tilde{u}_{r}-\iota_{2} \hat{\mathbf{W}}_{2}\right) \\
\dot{\hat{\delta}}_{2}=\xi_{2}\left(\tilde{u}_{r} h\left(\tilde{u}_{r}\right)-\lambda_{2}\left(\hat{\delta}_{2}-\delta_{2}^{0}\right)\right)
\end{array}\right.
\end{gathered}
$$

where $k_{3}$ and $k_{4}$ are positive design parameters; $k_{\zeta 1}$ and $k_{\zeta 2}$ are positive design parameters; $\hat{\delta}_{i}(i=1,2)$ is the estimation of $\delta_{i} ; h(\tilde{r})=\tanh \left(\tilde{r} / \chi_{r}\right), h\left(\tilde{u}_{r}\right)=\tanh \left(\tilde{u}_{r} / \chi_{u}\right), \chi_{j}(j=r, u)$ is a positive constant; $\boldsymbol{\Gamma}_{i}(i=1,2)$ is a positive define design matrix; $\lambda_{i}(i=1,2)$ is a small design parameter; $\delta_{j}^{0}(j=r, u)$ is the prior estimation of $\delta_{j}$; and $\sigma_{i}(i=1,2)$ is the state of the auxiliary system.

To compensate for the constraint effects of input saturation, auxiliary dynamic systems [28] are given as follows

$$
\dot{\sigma}_{1}= \begin{cases}-k_{\sigma_{1}} \sigma_{1}-\frac{\left|\tilde{r} \Delta \tau_{r}\right|+0.5 \Delta \tau_{r}^{2}}{\sigma_{1}^{2}} \sigma_{1}+\Delta \tau_{r}, & \left|\sigma_{1}\right| \geqslant \mu_{1} \\ 0, & \left|\sigma_{1}\right|<\mu_{1}\end{cases}
$$

and

$$
\dot{\sigma}_{2}= \begin{cases}-k_{\sigma 2} \sigma_{2}-\frac{\left|\tilde{u}_{r} \Delta \tau_{u}\right|+0.5 \Delta \tau_{u}^{2}}{\sigma_{2}^{2}} \sigma_{2}+\Delta \tau_{u}, & \left|\sigma_{2}\right| \geqslant \mu_{2} \\ 0, & \left|\sigma_{2}\right|<\mu_{2}\end{cases}
$$

where $k_{\sigma_{i}}(i=1,2)$ are positive design parameters; $\mu_{i}(i=1,2)$ are small positive constants; $\Delta \tau_{r}=$ $\tau_{r}-\tau_{r 0} ; \Delta \tau_{u}=\tau_{u}-\tau_{u 0}$.

Therefore, the closed-loop attitude and surge velocities tracking errors dynamics become by virtue of Equations (25) to (27)

$$
\left\{\begin{aligned}
m_{33} \dot{\tilde{r}} & =-k_{3} \tilde{r}-\tilde{\psi}-\tilde{\mathbf{W}}_{1} \boldsymbol{\varphi}(\mathbf{z})-\hat{\delta}_{1} h(\tilde{r})+k_{\varsigma 1} \sigma_{1}+\varepsilon_{1}+\tau_{w r}+\Delta \tau_{r} . \\
m_{11} \dot{u}_{e} & =-k_{4} u_{e}-\tilde{\mathbf{W}}_{2} \boldsymbol{\varphi}(\mathbf{z})-\hat{\delta}_{2} h\left(u_{e}\right)+k_{\varsigma^{2}} \sigma_{2}+\varepsilon_{2}+\tau_{w u}+\Delta \tau_{u} .
\end{aligned}\right.
$$

where $\tilde{\mathbf{W}}_{i}=\hat{\mathbf{W}}_{i}-\mathbf{W}_{i}^{*}(i=1,2)$ are weight matrix estimation errors; and $\tilde{\delta}_{i}=\hat{\delta}_{i}-\delta_{i}(i=1,2)$ are the adaptive terms estimation errors.

\subsection{Stability Analysis}

In this section, the main theorem of the path following controller is presented.

Theorem 2. Consider the USV model in Equations (4) and (5) in the presence of uncertainties and unknown external time-varying disturbances under input saturation, and suppose that Assumptions 1 and 2 are satisfied, under the guidance law in Equation (11) along with the finite-time currents observer in Equation (12). The given path is parameterized by $\theta$ with the update laws in Equation (21), and the desired velocity $u_{d}$ is given as well. The control laws in Equation (27) together with the adaptive laws in Equations (28) and (29) and the auxiliary 
systems in Equations (30) and (31) are incorporated to assist in handling input saturation, guaranteeing that all tracking error signals are locally UUВ.

Proof. Consider the following Lyapunov function

$$
V=\frac{1}{2} x_{e}^{2}+\frac{1}{2} y_{e}^{2}+\frac{1}{2} \tilde{\psi}^{2}+\frac{1}{2} m_{33} \tilde{r}^{2}+\frac{1}{2} m_{11} \tilde{u}_{r}^{2}+\frac{1}{2} \sum_{i=1}^{2} \sigma_{i}^{2}+\frac{1}{2} \sum_{i=1}^{2} \tilde{\mathbf{W}}_{i}^{T} \boldsymbol{\Gamma}_{i}^{-1} \tilde{\mathbf{W}}_{i}+\frac{1}{2 \tau_{i}} \sum_{i=1}^{2} \tilde{\delta}_{i}^{2}
$$

Taking the time derivative of Equation (33) along with Equations (22)-(24) and (32) yields

$$
\begin{aligned}
\dot{V} \leqslant & -k_{1} x_{e}^{2}-\frac{U_{r}}{\sqrt{\left(y_{e}+\alpha_{y}\right)^{2}+\Delta^{2}}} y_{e}^{2}-k_{2} \tilde{\psi}^{2}-k_{3} \tilde{r}^{2}-\tilde{\mathbf{W}}_{1} \boldsymbol{\varphi}(\mathbf{z}) \tilde{r}-\hat{\delta}_{1} h(\tilde{r}) \tilde{r} \\
& +k_{\zeta 1} \sigma_{1} \tilde{r}+\delta_{1} \tilde{r}+\Delta \tau_{r} \tilde{r}-k_{4} \tilde{u}_{r}^{2}-\tilde{\mathbf{W}}_{2} \boldsymbol{\varphi}(\mathbf{z}) \tilde{u}_{r}-\hat{\delta}_{2} h\left(\tilde{u}_{r}\right) \tilde{u}_{r}+k_{\zeta 2} \sigma_{2} \tilde{u}_{r}+\delta_{2} \tilde{u}_{r}+\Delta \tau_{u} \tilde{u}_{r} \\
& +\tilde{\theta}_{x} x_{e}+\tilde{\theta}_{y} y_{e}+\sum_{i=1}^{2} \sigma_{i} \dot{\sigma}_{i}+\sum_{i=1}^{2} \tilde{\mathbf{W}}_{i}^{T} \boldsymbol{\Gamma}_{i}^{-1} \dot{\mathbf{W}}_{i}+\frac{1}{\xi_{i}} \sum_{i=1}^{2} \tilde{\delta}_{i} \dot{\hat{\delta}}_{i}
\end{aligned}
$$

(1) When $\left|\sigma_{i}\right| \geqslant \mu_{i}(i=1,2)$, according to Equations (27) to (30) and Young's equalities $k_{\zeta 1} \sigma_{1} \tilde{r} \leqslant$ $\frac{1}{2} \tilde{r}^{2}+\frac{1}{2} k_{\zeta 1}^{2} \sigma_{1}^{2}, \sigma_{1} \Delta \tau_{r} \leqslant \frac{1}{2} \sigma_{1}^{2}+\frac{1}{2} \Delta \tau_{r}^{2}, k_{\zeta 2} \sigma_{2} \tilde{u}_{r} \leqslant \frac{1}{2} \tilde{u}_{r}^{2}+\frac{1}{2} k_{\zeta 2}^{2} \sigma_{2}^{2}, \sigma_{2} \Delta \tau_{u} \leqslant \frac{1}{2} \sigma_{2}^{2}+\frac{1}{2} \Delta \tau_{u}^{2}, \tilde{\theta}_{x} x_{e} \leqslant \frac{1}{2} \bar{\theta}^{2}+\frac{1}{2} x_{e}^{2}$, and $\tilde{\theta}_{y} y_{e} \leqslant \frac{k_{1}}{2} \bar{\theta}^{2}+\frac{1}{2 k_{1}} y_{e}^{2}$, we have

$$
\begin{aligned}
\dot{V} \leqslant & -\left(k_{1}-\frac{1}{2}\right) x_{e}^{2}-\left(\frac{U_{r}}{\sqrt{y_{e}^{2}+\Delta^{2}}}-\frac{1}{2 k_{1}}\right) y_{e}^{2}-k_{2} \tilde{\psi}^{2}-\left(k_{\sigma 1}-\frac{1}{2}-\frac{1}{2} k_{\zeta_{1}}^{2}\right) \sigma_{1}^{2}-\left(k_{3}-\frac{1}{2}\right) \tilde{r}^{2} \\
& -\left(k_{\sigma 2}-\frac{1}{2}-\frac{1}{2} k_{\zeta 2}^{2}\right) \sigma_{2}^{2}-\left(k_{4}-\frac{1}{2}\right) \tilde{u}_{r}^{2}-\sum_{i=1}^{2} \iota_{i} \tilde{\mathbf{W}}_{i}^{T} \hat{\mathbf{W}}_{i}-\sum_{i=1}^{2} \lambda_{i} \tilde{\delta}_{i}\left(\hat{\delta}_{i}-\delta_{i}^{0}\right) \\
& +\tilde{r}\left(\delta_{1}-\hat{\delta}_{1} h(\tilde{r})\right)+\tilde{\delta}_{1} h(\tilde{r}) \tilde{r}+\tilde{u}_{r}\left(\delta_{2}-\hat{\delta}_{2} h\left(\tilde{u}_{r}\right)\right)+\tilde{\delta}_{2} h\left(\tilde{u}_{r}\right) \tilde{u}_{r}+\frac{1+k_{1}}{2} \bar{\theta}^{2}
\end{aligned}
$$

Consider the following inequality of the hyperbolic tangent function holds for any $\chi>0$ and for any $\omega \in \mathbb{R}[29]$

$$
0 \leqslant|\omega|-\omega \tan \left(\frac{\mathscr{\omega}}{\chi}\right) \leqslant \kappa_{\chi} \chi
$$

where $\kappa_{\chi}$ is a constant that satisfies $\kappa_{\chi}=e^{-\left(\kappa_{\chi}+1\right)}$, i.e., $\kappa_{\chi}=0.2785$.

It is worth noting that the following equalities hold

$$
\begin{gathered}
-\sum_{i=1}^{2} \iota_{i} \tilde{\mathbf{W}}_{i}^{T} \hat{\mathbf{W}}_{i} \leqslant-\sum_{i=1}^{2} \frac{\iota_{i}}{2} \tilde{\mathbf{W}}_{i}^{T} \tilde{\mathbf{W}}_{\mathbf{i}}+\sum_{i=1}^{2} \frac{\iota_{i}}{2}\left\|\mathbf{W}_{i}^{*}\right\|^{2} \\
-\sum_{i=1}^{2} \lambda_{i} \tilde{\delta}_{i}\left(\hat{\delta}_{i}-\delta_{i}^{0}\right) \leqslant-\sum_{i=1}^{2} \frac{\lambda_{i}}{2} \tilde{\delta}_{i}^{2}+\sum_{i=1}^{2} \frac{\lambda_{i}}{2}\left(\delta_{i}-\delta_{i}^{0}\right)^{2} \\
\tilde{r}\left(\delta_{1}-\hat{\delta}_{1} \tan \left(\frac{\tilde{r}}{\chi_{r}}\right)\right)+\tilde{\delta}_{1} \tan \left(\frac{\tilde{r}}{\chi_{r}}\right) \tilde{r} \leqslant \delta_{1}\left(|\tilde{r}|-\tilde{r} \tan \left(\frac{\tilde{r}}{\chi_{r}}\right)\right) \leqslant 0.2785 \chi_{r} \delta_{1} \\
\tilde{u}_{r}\left(\delta_{2}-\hat{\delta}_{2} \tan \left(\frac{\tilde{u}_{r}}{\chi_{u}}\right)\right)+\tilde{\delta}_{2} \tan \left(\frac{\tilde{u}_{r}}{\chi_{u}}\right) \tilde{u}_{r} \leqslant \delta_{2}\left(\left|\tilde{u}_{r}\right|-\tilde{u}_{r} \tan \left(\frac{\tilde{u}_{r}}{\chi_{u}}\right)\right) \leqslant 0.2785 \chi_{u} \delta_{2}
\end{gathered}
$$


Substituting Equations (36)-(39) into Equation (35), we have

$$
\begin{aligned}
\dot{V} \leqslant & -\left(k_{1}-\frac{1}{2}\right) x_{e}^{2}-\left(\frac{U_{r}}{\sqrt{y_{e}^{2}+\Delta^{2}}}-\frac{1}{2 k_{1}}\right) y_{e}^{2}-k_{2} \tilde{\psi}^{2}-\left(k_{3}-\frac{1}{2}\right) \tilde{r}^{2}-\left(k_{4}-\frac{1}{2}\right) \tilde{u}_{r}^{2} \\
& -\sum_{i=1}^{2}\left(k_{\sigma i}-\frac{1}{2}-\frac{1}{2} k_{\zeta i}^{2}\right) \sigma_{i}^{2}-\sum_{i=1}^{2} \frac{\iota_{i}}{2} \tilde{\mathbf{W}}_{i}^{T} \tilde{\mathbf{W}}_{\mathbf{i}}-\sum_{i=1}^{2} \frac{\lambda_{i}}{2} \tilde{\delta}_{i}^{2}+\sum_{i=1}^{2} \frac{1}{2}\left(\delta_{i}-\delta_{i}^{0}\right)^{2} \\
& +\sum_{i=1}^{2} \frac{\iota_{i}}{2} W_{i M}{ }^{2}+0.2785\left(\chi_{r} \delta_{1}+\chi_{u} \delta_{2}\right)+\frac{1+k_{1}}{2} \bar{\theta}^{2} \\
\leqslant & -\kappa_{1} V+\vartheta_{1}
\end{aligned}
$$

where $\kappa_{1}=\min \left\{2 k_{1}-1, \frac{2 U_{r}}{\sqrt{y_{e}^{2}+\Delta^{2}}}-\frac{1}{k_{1}}, 2 k_{2}, \frac{2 k_{3}-1}{m_{33}}, \frac{2 k_{4}-1}{m_{11}}, \min _{i=1,2}\left(2 k_{\sigma i}-1-k_{\zeta i}^{2}\right), \min _{i=1,2}\left(l_{i} \lambda_{\min }\left(\boldsymbol{\Gamma}_{i}\right)\right), \min _{i=1,2}\left(\lambda_{i} \xi_{i}\right)\right\}$, $k_{1}=\max \left\{\frac{1}{2}, \frac{\sqrt{y_{e}^{2}+\Delta^{2}}}{2 U_{r}}\right\}, \lambda_{\min }(\bullet)$ denotes the minimum eigenvalue of a matrix, and $\vartheta_{1}=$ $\sum_{i=1}^{2} \frac{1}{2}\left(\delta_{i}-\delta_{i}^{0}\right)^{2}+\sum_{i=1}^{2} \frac{\iota_{i}}{2} W_{i M^{2}}+0.2785\left(\chi_{r} \delta_{1}+\chi_{u} \delta_{2}\right)+\frac{1+k_{1}}{2} \bar{\theta}^{2}$.

(2) When $\left|\sigma_{i}\right|<\mu_{i}(i=1,2)$, we have $\sum_{i=1}^{2} \sigma_{i} \dot{\sigma}_{i}=0$. According to Equations (30) and (31) and the inequalities $\sum_{i=1}^{2} \frac{1}{2} k_{\zeta i}^{2} \sigma_{i}^{2} \leqslant-\sum_{i=1}^{2} \frac{1}{2} k_{\zeta i}^{2} \sigma_{i}^{2}+\sum_{i=1}^{2} k_{\zeta i}^{2} \mu_{i}^{2}, \tilde{r} \Delta \tau_{r} \leqslant \frac{1}{2} \tilde{r}^{2}+\frac{1}{2} \Delta \tau_{r}^{2}$, and $\tilde{u}_{r} \Delta \tau_{u} \leqslant \frac{1}{2} \tilde{u}_{r}^{2}+\frac{1}{2} \Delta \tau_{u}^{2}$, Equation (34) becomes

$$
\begin{aligned}
\dot{V} \leqslant & -\left(k_{1}-\frac{1}{2}\right) x_{e}^{2}-\left(\frac{U_{r}}{\sqrt{y_{e}^{2}+\Delta^{2}}}-\frac{1}{2 k_{1}}\right) y_{e}^{2}-k_{2} \tilde{\psi}^{2}-\left(k_{3}-1\right) \tilde{r}^{2}-\left(k_{4}-1\right) \tilde{u}_{r}^{2} \\
& -\sum_{i=1}^{2} \frac{k_{\zeta i}^{2}}{2} \sigma_{i}^{2}-\sum_{i=1}^{2} \frac{\iota_{i}}{2} \tilde{\mathbf{W}}_{i}^{T} \tilde{\mathbf{W}}_{i}-\sum_{i=1}^{2} \frac{\lambda_{i}}{2} \tilde{\delta}_{i}^{2}+\sum_{i=1}^{2} \frac{1}{2}\left(\delta_{i}-\delta_{i}^{0}\right)^{2}+\sum_{i=1}^{2} \frac{\iota_{i}}{2} W_{i M}{ }^{2} \\
& +0.2785\left(\chi_{r} \delta_{1}+\chi_{u} \delta_{2}\right)+\sum_{i=1}^{2} k_{\zeta i}^{2} \mu_{i}^{2}+\frac{1}{2} \Delta \tau_{r}^{2}+\frac{1}{2} \Delta \tau_{u}^{2}+\frac{1+k_{1}}{2} \bar{\theta}^{2} \\
\leqslant & -\kappa_{2} V+\vartheta_{2}
\end{aligned}
$$

where $\kappa_{2}=\min \left\{2 k_{1}-1, \frac{2 U_{r}}{\sqrt{y_{e}^{2}+\Delta^{2}}}-\frac{1}{k_{1}}, 2 k_{2}, \frac{2 k_{3}-2}{m_{33}}, \frac{2 k_{4}-2}{m_{11}}, \min _{i=1,2}\left(k_{\zeta i}^{2}\right), \min _{i=1,2}\left(\iota_{i} \lambda_{\min }\left(\boldsymbol{\Gamma}_{i}\right)\right), \min _{i=1,2}\left(\lambda_{i} \xi_{i}\right)\right\}$, $k_{1}=\max \left\{\frac{1}{2}, \frac{\sqrt{y_{e}^{2}+\Delta^{2}}}{2 u_{r}}\right\} ; \vartheta_{2}=\sum_{i=1}^{2} \frac{1}{2}\left(\delta_{i}-\delta_{i}^{0}\right)^{2}+\sum_{i=1}^{2} \frac{\iota_{i}}{2} W_{i M^{2}}+0.2785\left(\chi_{r} \delta_{1}+\chi_{u} \delta_{2}\right)+\sum_{i=1}^{2} k_{\zeta i}^{2} \mu_{i}^{2}+\frac{1}{2} \Delta \tau_{r}^{2}+$ $\frac{1}{2} \Delta \tau_{u}^{2}+\frac{1+k_{1}}{2} \bar{\theta}^{2}$.

Synthesizing Equations (41) and (42), we have

$$
\dot{V} \leqslant-\kappa V+\vartheta
$$

where $\kappa=\min \left\{\kappa_{1}, \kappa_{2}\right\}$ and $\vartheta=\max \left\{\vartheta_{1}, \vartheta_{2}\right\}$ with the design parameters satisfying the conditions: $k_{1}>\max \left(\frac{1}{2}, \frac{\sqrt{y_{e}^{2}+\Delta^{2}}}{2 U_{r}}\right), k_{2}>0, k_{3}>1, k_{4}>1, k_{\zeta 1}>0, k_{\zeta 2}>0, k_{\sigma 1}>\frac{1}{2} k_{\zeta 1}^{2}+\frac{1}{2}, k_{\sigma 2}>\frac{1}{2} k_{\zeta 2}^{2}+\frac{1}{2}$. Then, the following inequality can be obtained

$$
0 \leqslant V \leqslant\left(V(0)-\frac{\vartheta}{\kappa}\right) e^{-\kappa t}+\frac{\vartheta}{\kappa}
$$

In conclusion, it follows the definition of $V$ that the tracking error signals $\eta_{e}=$ $\left[x_{e}, y_{e}, \tilde{\psi}, \tilde{r}, \tilde{u}_{r}\right]^{T}$ are locally UUB, which ultimately converges to the compact sets $\Omega_{1}=$ $\left\{\eta_{e} \in \mathbb{R}^{5} \mid\left\|\eta_{e}\right\| \leqslant \sqrt{2\left(V(0)-\frac{\vartheta}{\kappa}\right) e^{-\kappa t}+\frac{2 \vartheta}{\kappa}}\right\}$. The ultimate compact set can be easily tuned by 
adjusting the design parameters. Meanwhile, the parameters estimation errors $\tilde{\mathbf{W}}_{1}, \tilde{\mathbf{W}}_{2}, \tilde{\delta}_{1}$, and $\tilde{\delta}_{2}$ are locally UUB. Theorem 2 is thus proved.

\subsection{Sway Dynamics}

For the sway velocity dynamics, considering the Lyapunov function $V_{v}=\frac{1}{2} v_{r}^{2}$ and taking the time derivative of it based on Equation (5) yields

$$
\begin{aligned}
\dot{V}_{v} & =v_{r} \dot{v}_{r} \\
& =-\frac{m_{11}}{m_{22}} u_{r} r v_{r}-\frac{d_{22}}{m_{22}} v_{r}^{2}-\frac{d_{v 2}}{m_{11}}\left|v_{r}\right| v_{r}^{2}-\frac{d_{v 3}}{m_{11}} v_{r}^{4}+\frac{1}{m_{22}} \tau_{w v} \\
& \leqslant-\chi v_{r}+\frac{1}{m_{22}} \bar{\tau}_{w}
\end{aligned}
$$

where $\chi=\frac{m_{11}}{m_{22}} u_{r} r$. Based on the above analyses, the boundedness of $\tilde{u}_{r}$ and $\tilde{r}$ is guaranteed, thus $\chi$ is bounded. It should be noted that $d_{v i}$ and $m_{i i}(i=2,3)$ are positive constants. According to Krstic, M.; Kanellakopoulos, I.; Kokotovic, P. V. [30], we have

$$
\begin{aligned}
V_{v} & \leqslant V_{v}\left(t_{0}\right) e^{-\chi\left(t-t_{0}\right)}+\bar{\tau}_{w} \frac{1-e^{-\chi\left(t-t_{0}\right)}}{m_{22} \chi} \\
& \leqslant V_{v}\left(t_{0}\right)+\frac{\bar{\tau}_{w}}{m_{22} \chi}
\end{aligned}
$$

Therefore, the boundedness of the sway velocity $v_{r}$ is guaranteed.

\section{Simulations}

The effectiveness and robustness of the proposed path following control method were evaluated based on the platform of MATLAB. The Cybership II [31] was taken as the control object whose parameters were as follows: $m_{11}=25.8, m_{22}=33.8, m_{33}=2.76, d_{11}=0.9257, d_{22}=2.8909, d_{33}=0.5$. For simplicity, we ignored the off-diagonal terms of the inertia and damping. The maximum actuated force and moment were $2 \mathrm{~N}$ and $1.5 \mathrm{Nm}$. From the beginning of the simulations, the ocean currents with time-varying speed were given as $V_{x}=0.08 \sin (0.1 t) \mathrm{m} / \mathrm{s}$ and $V_{y}=0.04 \sin (0.1 t) \mathrm{m} / \mathrm{s}$. The time-varying external disturbances were generated with the first-order Markov process $\dot{\tau}_{w u}+\varsigma_{1} \tau_{w u}=w_{1}, \dot{\tau}_{w v}+\varsigma_{2} \tau_{w v}=w_{2}, \dot{\tau}_{w r}+\varsigma_{3} \tau_{w r}=w_{3}$, where $w_{i}$ and $\varsigma_{i}(i=1,2,3)$ are zero-mean Gaussian white noise and constants, respectively [32]. The parameters of the controller are listed in Table 1. The node numbers and widths of RBFNNs were chosen as: node number $l=21$ and the widths $b_{i}=3(i=1,2, \ldots, l)$. The neural active region was chosen as $[|u|,|v|,|r|] \in$ $[[0,2],[0,1.5],[0,1.5]]^{T}$. Performance comparisons between the proposed finite-time currents observer based ILOS guidance with adaptive RBFNN (FCONN) controller and the indirect adaptive observer based [10] ILOS guidance with adaptive RBFNN (IAONN) controller are presented in the following two control scenarios.

Table 1. Parameters of the path following controller.

\begin{tabular}{cccccccccc}
\hline Notation & Value & Natation & Value & Natation & Value & Natation & Value & Natation & Value \\
\hline$k_{1}$ & 1 & $l_{2}$ & 4 & $\Gamma_{1} i i(i=21)$ & 500 & $\xi_{2}$ & 20 & $\chi_{r}$ & 0.01 \\
$k_{2}$ & 2 & $\rho_{1}$ & 0.01 & $\Gamma_{2} i i(i=21)$ & 50 & $\lambda_{1}$ & 0.01 & $\chi_{u}$ & 1 \\
$k_{3}$ & 4 & $\rho_{2}$ & 0.03 & $\iota_{1}$ & 0.05 & $\lambda_{2}$ & 0.01 & $\Delta$ & 2.51 \\
$k_{4}$ & 5 & $k_{\zeta_{1}}$ & 1.2 & $\iota_{2}$ & 0.05 & $\delta_{1}^{0}$ & 0.1 & & \\
$l_{1}$ & 100 & $k_{\zeta_{2}}$ & 1.2 & $\xi_{1}$ & 50 & $\delta_{1}^{0}$ & 0.1 & & \\
\hline
\end{tabular}

Case 1: The desired path and speed assignment were chosen as $\boldsymbol{P}_{d}=[\theta, \theta]^{T}$ and $u_{d}=0.5 \mathrm{~m} / \mathrm{s}$. The initial states were given as $\left[x(0), y(0), u_{r}(0), v_{r}(0), r\right]^{T}=[0 \mathrm{~m}, 2 \mathrm{~m}, 0.01 \mathrm{~m} / \mathrm{s}, 0 \mathrm{~m} / \mathrm{s}, 0 \mathrm{rad} / \mathrm{s}]^{T}$. 
The simulation results are shown in Figure $3 \mathrm{a}-\mathrm{g}$. Table 2 summarizes the performance indices based on the integrated absolute error (IAE) and the time integrated absolute error (ITAE), which were used to evaluate the transient performance and steady-state performance. The proposed FCONN control method could drive the USV following the desired path with a high-precision and better transient process (Figure 3a). Their detailed distinction is more clearly shown in Figure 3b,c, specifically the smaller along- and cross-tracking errors and the smaller heading and surge velocity tracking errors. Meanwhile, the lower IAE and ITAE metrical values of the cross-tracking error revealed the better transient and steady-state performance. Figure $3 \mathrm{~d}$ shows that the proposed finite-time currents observer could identify the time-varying currents accurately, whereas the IAONN control scheme had an obvious oscillation during the transient process, as well as lower accuracy in the steady state. Figure 3d demonstrates that the RBFNNs could capture the unknown dynamical uncertainties precisely and Figure $3 e$ presents the compound bounds and their estimation, which played decisive roles in driving the dynamics state $r$ and $u_{r}$ to their real value. Figure $3 \mathrm{f}$ depicts the profile of the control inputs where the input saturation (IS) problem Was effectively compensated by the auxiliary system. The control inputs of the proposed method were in the specified region. Hence, in the case, the proposed FCONN path following control method was more effective and robust according to these simulation results.

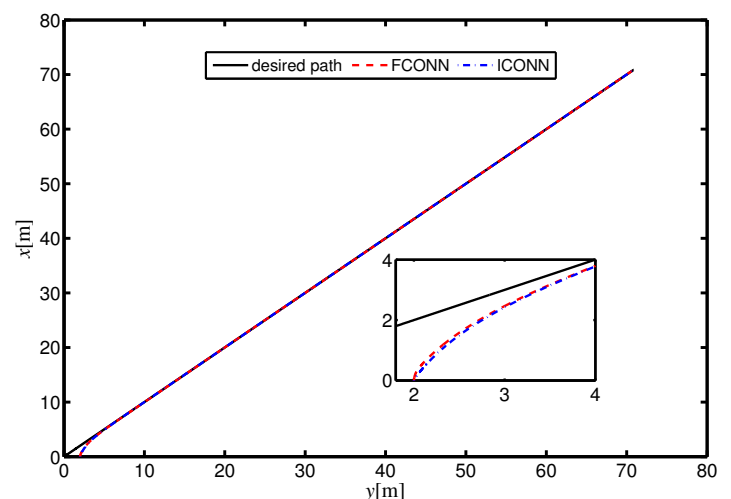

(a) line path following.
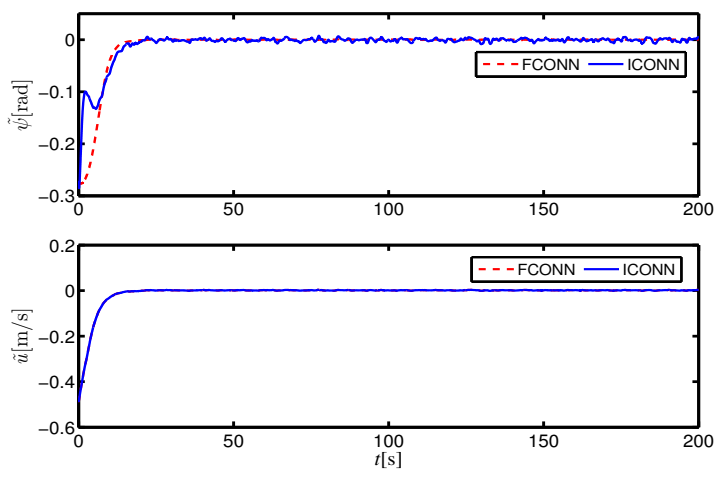

(c) Profile of the heading and surge velocity tracking in Case 1.
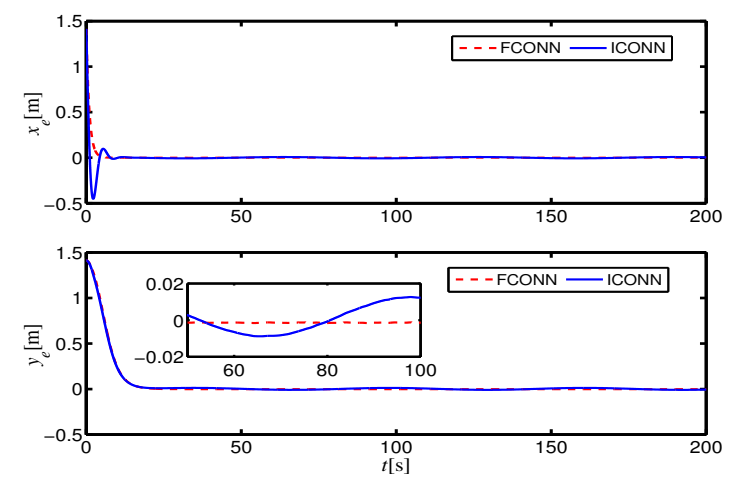

(b) Along- and cross-tracking error in Case 1.
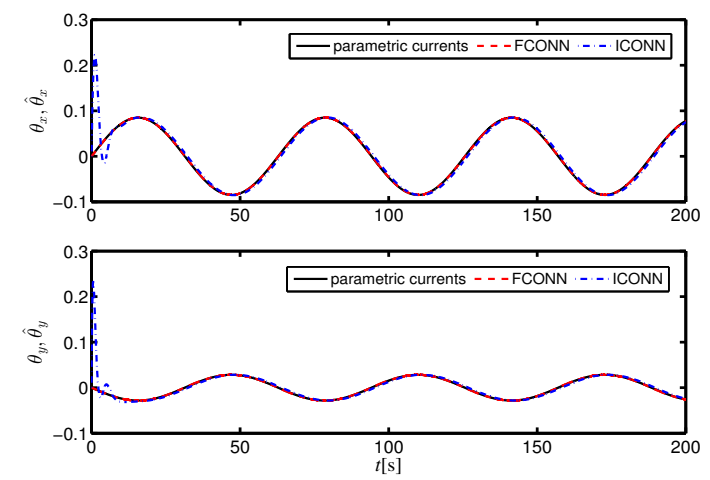

(d) Estimations of parametric currents in Case 1.

Figure 3. Cont. 

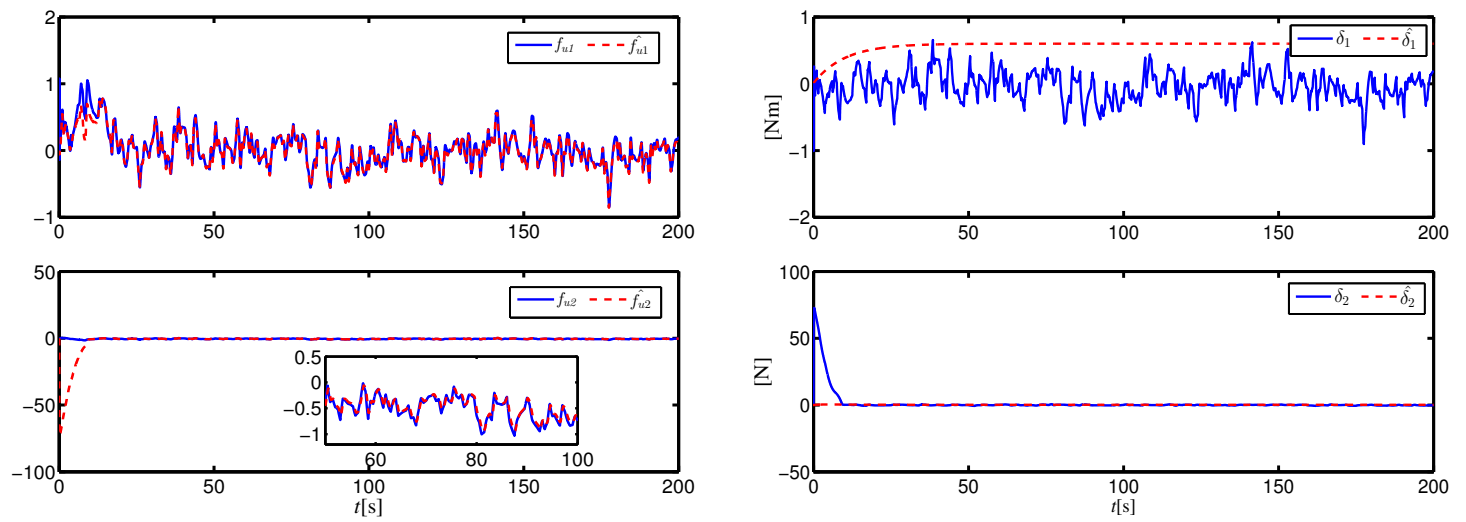

(e) Estimations of dynamical uncertainties using RBFNN in Case 1.

(f) Estimations of compound bound in Case 1.
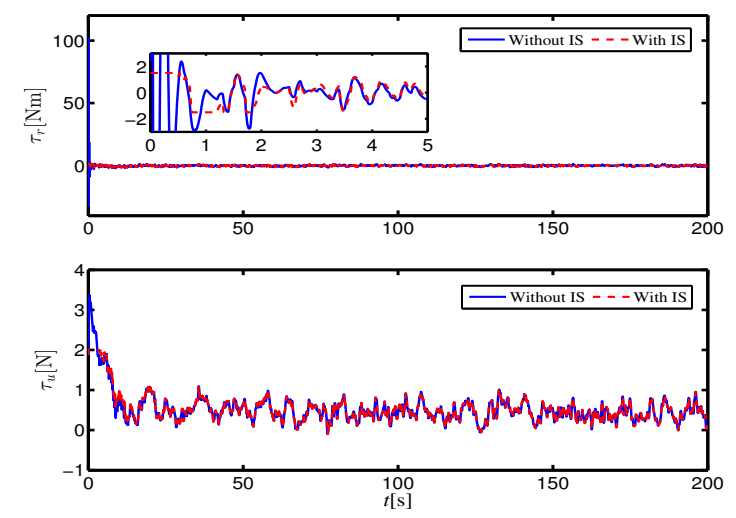

(g) Profile of the control inputs in Case 1.

Figure 3. Line path following results.

Table 2. Performance indices in these two path following scenarios.

\begin{tabular}{|c|c|c|c|c|}
\hline \multirow[b]{2}{*}{ Control Law } & \multicolumn{2}{|c|}{ Line Path Following } & \multicolumn{2}{|c|}{ Curvilinear Path Following } \\
\hline & $\begin{array}{l}\text { IAE }\left(\cdot 10^{3}\right) \\
\int_{0}^{t}\left|y_{e}\right| d \tau\end{array}$ & $\begin{array}{r}\operatorname{ITAE}\left(\cdot 10^{3}\right) \\
\int_{0}^{t} t\left|y_{e}\right| d \tau\end{array}$ & $\begin{array}{l}\operatorname{IAE}\left(\cdot 10^{4}\right) \\
\int_{0}^{t}\left|y_{e}\right| d \tau\end{array}$ & $\begin{array}{l}\text { ITAE }\left(\cdot 10^{4}\right) \\
\int_{0}^{t} t\left|y_{e}\right| d \tau\end{array}$ \\
\hline FCONN & 1.92 & 5.78 & 2.58 & 9.01 \\
\hline IAONN & 4.18 & 13.12 & 7.32 & 20.32 \\
\hline
\end{tabular}

Case 2: Similar to Case 1, another comparison is presented to verify the performance in the case of following a curvilinear path with the same design parameters. The desired path and speed assignment were chosen as $\boldsymbol{P}_{d}=\theta,[10 \sin (0.1 \theta)]^{T}$ and $u_{d}=0.5 \mathrm{~m} / \mathrm{s}$. The initial states were given as $\left[x(0), y(0), u_{r}(0), v_{r}(0), r\right]^{T}=[0 \mathrm{~m}, 2 \mathrm{~m}, 0.01 \mathrm{~m} / \mathrm{s}, 0 \mathrm{~m} / \mathrm{s}, 0 \mathrm{rad} / \mathrm{s}]^{T}$. The simulations results are shown in Figure $4 \mathrm{a}-\mathrm{g}$ and the performance quantification indices are summarized in Table 2. As illustrated in Figure $4 \mathrm{a}$, although following the curvilinear path, the proposed FCONN method behaved almost the same in both control scenarios. As shown in Figure $4 \mathrm{~b}$, the along- and cross-tracking errors oscillated to varying degrees for the poor performance of IAONN, whereas the position errors of FCONN could smoothly and steadily converge to a small neighborhood around zero within a short time. Moreover, the smaller IAE and ITAE metrical values verified it. Figure $4 \mathrm{c}$ shows the slight oscillation of the heading and surge velocity tracking error of IAONN. Moreover, in Figure $4 \mathrm{~d}$, the poor performance of estimating ocean currents of IAONN undoubtedly degraded the tracking performance. Figure $4 \mathrm{e}, \mathrm{f}$ shows the exceptional performance of the dynamical uncertainties estimation and disturbance rejection 
of the FCONN method. In addition, the control inputs were in the specified region for introducing the auxiliary system, as depicted in Figure 4g. Overall, the proposed control method achieved satisfactory performance and robustness in both cases with fast and accurate estimations of the unknown time-varying ocean currents and dynamical uncertainties, and satisfactory rejection of external disturbances.

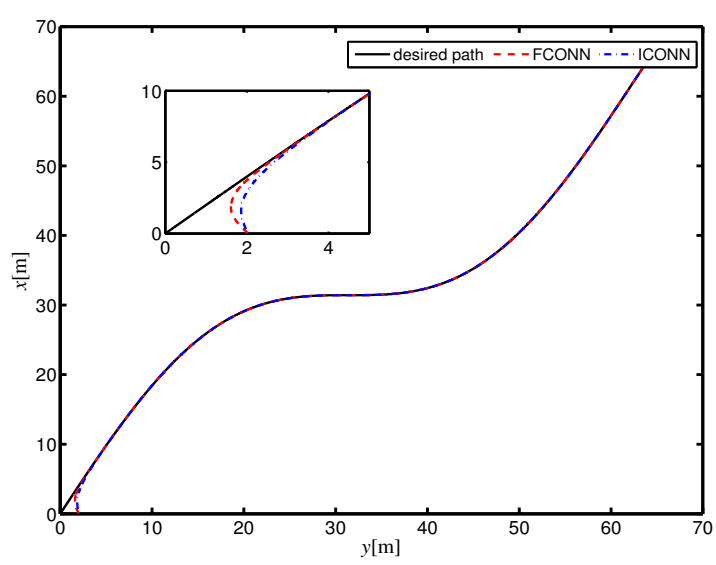

(a) Curvilinear path following.
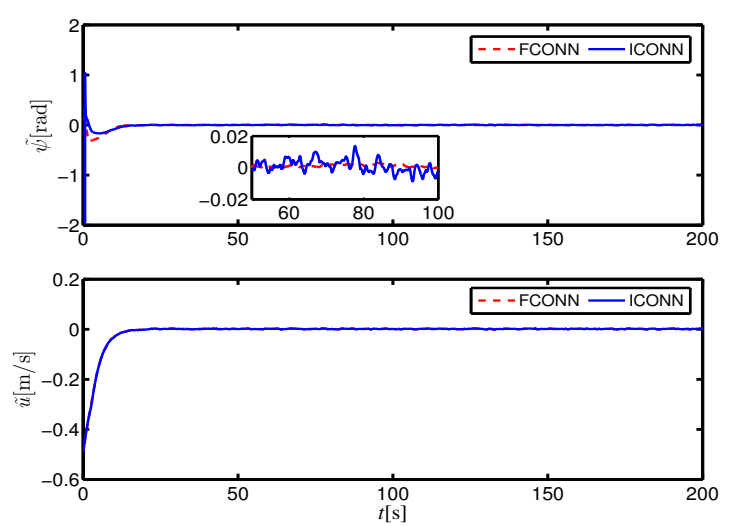

(c) Profile of the heading and surge velocity tracking in Case 2.
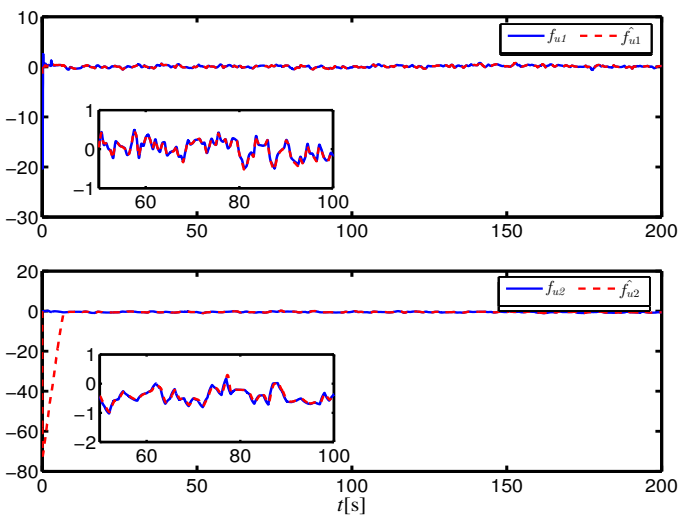

(e) Estimations of dynamical uncertainties using RBFNN in Case 2.
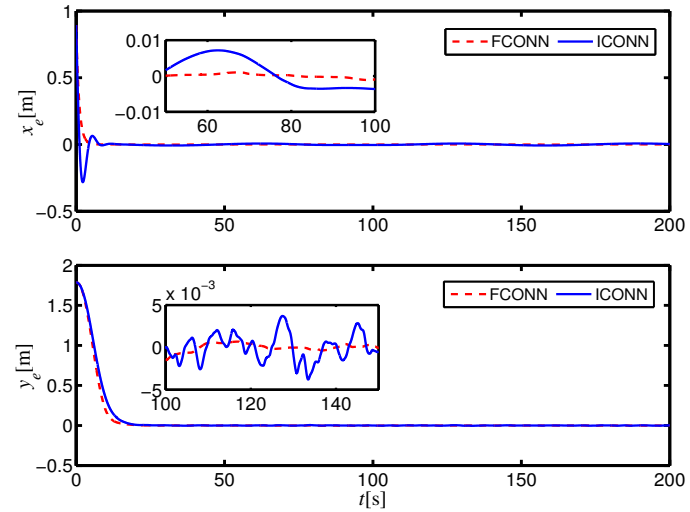

(b) Along- and cross-tracking error in Case 2.
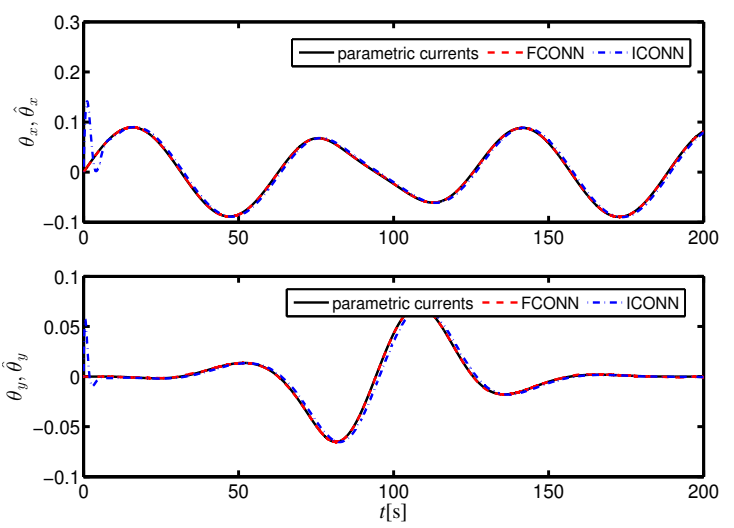

(d) Estimations of parametric currents in Case 2.
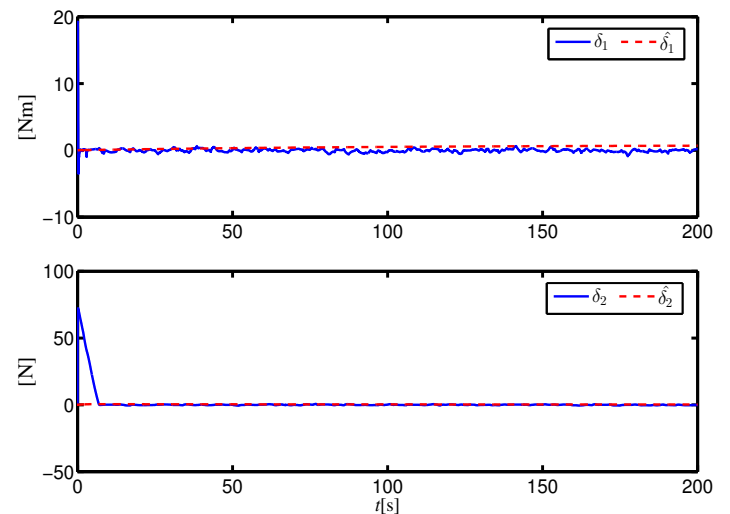

(f) Estimations of compound bound in Case 2.

Figure 4. Cont. 

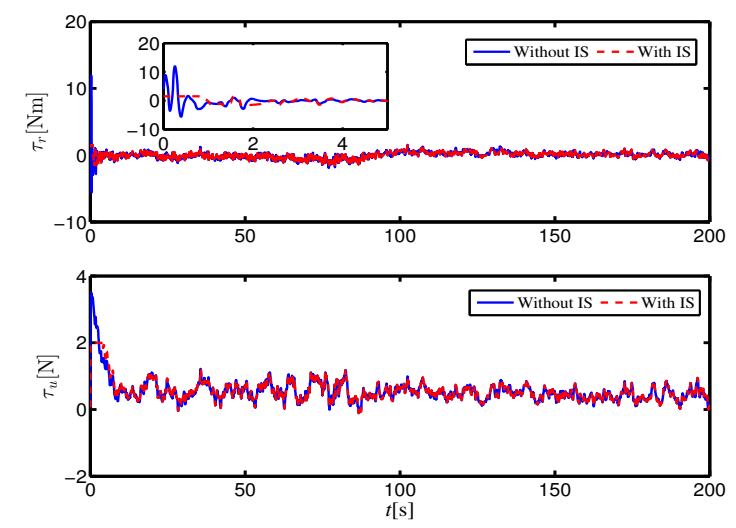

(g) Profile of the control inputs in Case 2.

Figure 4. Curvilinear path following results.

\section{Conclusions}

In this paper, a path following control scheme for a USV subject to input saturation and uncertainties has been proposed by resorting to the finite-time currents observer based ILOS guidance, the adaptive RBFNN, and the auxiliary dynamic system. The finite-time currents observer based ILOS guidance is applied to obtain the desired yaw angle, where the incorporated finite time currents observer can provide the precise estimations of the unknown time-varying ocean currents. Simultaneously, the RBF neural networks and the adaptive laws with leakages terms can provide the precise estimations of dynamical uncertainties and the compound bounds of the approximation errors and external disturbances, without knowing any prior knowledge of the time-varying disturbance. The auxiliary control system is introduced to handle input saturation of the actuators. It has been proved that all error signals of the closed-loop system are locally UUB. Finally, both linear and curved path following are presented and compared with the preceding control method. Simulations results have verified that the proposed control method can achieve satisfactory performance and robustness. Future work will cover the aspect of the position error constraint to ensure that the USV can work in these situations including the narrow passage and the channel between obstacles, as well as the precise estimation of the sideslip angle.

Author Contributions: Y.F., H.H. and Y.T. conceived the framework and wrote the paper. Y.F. and H.H. designed the controller and H.H. and Y.T. were responsible for the formula analyses. H.H. made the simulations and Y.T. analyzed the data. Y.F., H.H. and Y.T. discussed the results and contributed to the whole manuscript.

Funding: This research was funded by National Nature Science Foundation under grant number 51609033, in part by Natural Science Foundation of Liaoning Province under Grant number 201801732, and in part by Fundamental Research Funds for the Central Universities under Grant number 3132016312.

Conflicts of Interest: The authors declare no conflict of interest.

\section{References}

1. Liu, Z.; Zhang, Y.; Yu, X.; Yuan, C. Unmanned surface vehicles: An overview of developments and challenges. Annu. Rev. Control. 2016, 41, 71-93. [CrossRef]

2. Ashrafiuon, H.; Muske, K.R.; Mcninch, L.C. Review of nonlinear tracking and setpoint control approaches for autonomous underactuated marine vehicles. In Proceedings of the 2010 American Control Conference, Baltimore, MD, USA, 30 June-2 July 2010; pp. 5203-5211.

3. Do, K.D.; Pan, J. Control of ships and underwater vehicles. In Advances in Industrial Control; Springer: London, UK, 2009; pp. 40-42, ISBN 978-1-84882-729-5.

4. Caccia, M.; Bibuli, M.; Bono, R.; Bruzzone, G. Basic navigation, guidance and control of an unmanned surface vehicle. Auton. Robot. 2008, 25, 349-365. [CrossRef] 
5. Breivik, M.; Fossen, T.I. Path following for marine surface vessels. In Proceedings of the Oceans '04 MTS/IEEE Techno-Ocean '04, Kobe, Japan, 9-12 November 2004; pp. 2282-2289.

6. Fossen, T.I.; Breivik, M.; Skjetne, R. Line-of-sight path following of underactuated marine craft. In Proceedings of the IFAC Manoeuvring and control of Marine Craft, Girona, Spain, 17-19 September 2003; pp. 211-216.

7. Fossen, T.I.; Pettersen, K.Y. On uniform semiglobal exponential stability (usges) of proportional line-of-sight guidance laws. Automatica 2014, 50, 2912-2917. [CrossRef]

8. Borhaug, E.; Pavlov, A.; Pettersen, K.Y. Integral LOS control for path following of underactuated marine surface vessels in the presence of constant ocean currents. In Proceedings of the 47th IEEE conference on Decision and Control, Cancun, Mexico, 9-11 September 2008; pp. 4984-4991.

9. Lekkas, A.M.; Fossen, T.I. Integral los path following for curved paths based on a monotone cubic hermite spline parametrization. IEEE Trans. Control. Syst. Technol. 2014, 22, 2287-2301. [CrossRef]

10. Fossen, T.I.; Lekkas, A.M. Direct and indirect adaptive integral line-of-sight path-following controllers for marine craft exposed to ocean currents. Int. J. Adapt. Control. Signal Process. 2017, 31, 445-463. [CrossRef]

11. Fossen, T.I.; Pettersen, K.Y.; Galeazzi, R. Line-of-sight path following for dubins paths with adaptive sideslip compensation of drift forces. IEEE Trans. Control. Syst. Technol. 2015, 23, 820-827. [CrossRef]

12. Liu, L.; Wang, D.; Peng, Z. Eso-based line-of-sight guidance law for path following of underactuated marine surface vehicles with exact sideslip compensation. IEEE J. Ocean. Eng. 2017, 42, 477-487. [CrossRef]

13. Wang, N.; Sun, Z.; Yin, J.; Su, S.F.; Sharma, S. Finite-time observer based guidance and control of underactuated surface vehicles with unknown sideslip angles and disturbances. IEEE Access 2018, 6, 14059-14070. [CrossRef]

14. Wang, W.; Huang, J.; Wen, C.; Fan, H. Distributed adaptive control for consensus tracking with application to formation control of nonholonomic mobile robots. Automatica 2014, 50, 1254-1263. [CrossRef]

15. Miao, J.; Wang, S.; Tomovic, M.M.; Zhao, Z. Compound line-of-sight nonlinear path following control of underactuated marine vehicles exposed to wind, waves, and ocean currents. Nonlinear Dyn. 2017, 89, 1-19. [CrossRef]

16. Do, K.D. Global robust adaptive path-tracking control of underactuated ships under stochastic disturbances. Ocean. Eng. 2016, 111, 267-278. [CrossRef]

17. Liu, S.; Liu, Y.; Wang, N. Nonlinear disturbance observer-based backstepping finite-time sliding mode tracking control of underwater vehicles with system uncertainties and external disturbances. Nonlinear Dyn. 2017, 88, 1-12. [CrossRef]

18. Du, J.; Hu, X.; Krstić, M.; Sun, Y. Robust dynamic positioning of ships with disturbances under input saturation. Automatica 2016, 73, 207-214. [CrossRef]

19. Sun, Z.; Zhang, G.; Yi, B.; Zhang, W. Practical proportional integral sliding mode control for underactuated surface ships in the fields of marine practice. Ocean. Eng. 2017, 142, 217-223. [CrossRef]

20. Roy, S.; Roy, S.B.; Kar, I.N. Adaptive-robust control of euler-lagrange systems with linearly parametrizable uncertainty bound. IEEE Trans. Control. Syst. Technol. 2017, 26, 1842-1850. [CrossRef]

21. Roy, S.; Roy, S.B.; Kar, I.N. A New Design Methodology of Adaptive Sliding Mode Control for a Class of Nonlinear Systems with State Dependent Uncertainty Bound. In Proceedings of the 15th International Workshop on Variable Structure Systems, Graz, Austria, 9-11 September 2018; pp. 414-419.

22. Wang, N.; Er, M.J.; Sun, J.C.; Liu, Y.C. Adaptive robust online constructive fuzzy control of a complex surface vehicle system. IEEE Trans. Cybern. 2017, 46, 1511-1523. [CrossRef]

23. Zheng, Z.; Sun, L. Path following control for marine surface vessel with uncertainties and input saturation. Neurocomputing 2016, 177, 158-167. [CrossRef]

24. Liu, L.; Wang, D.; Peng, Z. Path following of marine surface vehicles with dynamical uncertainty and time-varying ocean disturbances. Neurocomputing 2016, 173, 799-808. [CrossRef]

25. Zheng, Z.; Feroskhan, M. Path following of a surface vessel with prescribed performance in the presence of input saturation and external disturbances. IEEE/ASME Trans. Mechatronics 2017, 22, 2564-2575. [CrossRef]

26. Roy, S.; Shome, S.N.; Nandy, S.; Ray, R.; Kumar, V. Trajectory following control of auv: A robust approach. J. Inst. Eng. India Series C 2013, 94, 253-265. [CrossRef]

27. Shtessel, Y.B.; Shkolnikov, I.A.; Levant, A. Smooth second-order sliding modes: Missile guidance application. Automatica 2007, 43, 1470-1476. [CrossRef] 
28. Chen, M.; Ge, S.S.; Ren, B. Adaptive tracking control of uncertain mimo nonlinear systems with input constraints. Automatica 2011, 47, 452-465. [CrossRef]

29. Polycarpou, M.M.; Ioannou, P.A. A robust adaptive nonlinear control design. Automatica 1996, 32, $423-427$. [CrossRef]

30. Krstic, M.; Kanellakopoulos, I.; Kokotovic, P.V. Nonlinear and adaptive control. In Lecture Notes in Control and Information; Sciences: New York, NY, USA, 1995; pp. 511-514, ISBN 9780471127321.

31. Fredriksen, E.; Pettersen, K.Y. Global k-exponential way-point maneuvering of ships: Theory and experiments. Automatica 2009, 42, 677-687. [CrossRef]

32. Fossen, T.I. How to incorporate wind, waves and ocean currents in the marine craft equations of motion. In Proceedings of the 9th IFAC Conference on Manoeuvring and Control of Marine Craft, Arenzano, Italy, 19-21 September 2012; pp. 126-131.

(C) 2019 by the authors. Licensee MDPI, Basel, Switzerland. This article is an open access article distributed under the terms and conditions of the Creative Commons Attribution (CC BY) license (http://creativecommons.org/licenses/by/4.0/). 